\title{
Accelerated robust optimization algorithm for proton therapy treatment planning.
}

\author{
Gregory Buti ${ }^{1}$, Kevin Souris ${ }^{1}$, Ana M. Barragán Montero ${ }^{1}$, Marie \\ Cohilis ${ }^{1}$, John A. Lee ${ }^{1}$, Edmond Sterpin ${ }^{1,2}$ \\ ${ }^{1}$ Université Catholique de Louvain, Institut de Recherche Expérimentale et Clinique (IREC), \\ Center of Molecular Imaging, Radiotherapy and Oncology (MIRO), Brussels, Belgium \\ ${ }^{2}$ Katholieke Universiteit Leuven, Department of Oncology, Laboratory of Experimental \\ Radiotherapy, Leuven, Belgium
}

Version typeset February 12, 2020

E-mail: gregory.buti@uclouvain.be

\begin{abstract}
Purpose: Robust optimization is a computational expensive process resulting in long plan computation times. This issue is especially critical for moving targets as these need a large number of uncertainty scenarios to robustly optimize their treatment plans. In this study, we propose a novel worst-case robust optimization algorithm, called dynamic minimax, that accelerates the conventional minimax optimization. Dynamic minimax optimization aims at speeding up the plan optimization process by decreasing the number of evaluated scenarios in the optimization.

Methods: For a given pool of scenarios (for instance $63=7$ setup $\times 3$ range $\times 3$ breathing phases), the proposed dynamic minimax algorithm only considers a reduced number of candidate-worst scenarios, selected from the full 63 scenario set. These scenarios are updated throughout the optimization by randomly sampling new scenarios according to a hidden variable $P$, called the 'probability acceptance function', which associates with each scenario the probability of it being selected as the worst case. By doing so, the algorithm favors scenarios that are mostly "active", that is, frequently evaluated as the worst case. Additionally, unconsidered scenarios have the possibility to be re-considered, later on in the optimization, depending on the convergence towards a particular solution.

The proposed algorithm was implemented in the open-source robust optimizer MIROpt and tested for six 4D-IMPT lung tumor patients with various tumor sizes
\end{abstract}


and motions. Treatment plans were evaluated by performing comprehensive robustness tests (simulating range errors, systematic setup errors and breathing motion) using the open-source Monte-Carlo dose engine MCsquare.

Results: The dynamic minimax algorithm achieved an optimization time gain of $84 \%$, on average. The dynamic minimax optimization results in a significantly noisier optimization process due to the fact that more scenarios are accessed in the optimization. However, the increased noise level does not harm the final quality of the plan. In fact, the plan quality is similar between dynamic and conventional minimax optimization with regards to target coverage and normal tissue sparing: on average, the difference in worst-case D95 is 0.2 Gy and the difference in mean lung dose and mean heart dose is 0.4 Gy and 0.1 Gy, respectively (evaluated in the nominal scenario).

Conclusions: The proposed worst-case 4D-robust optimization algorithm achieves a significant optimization time gain of $84 \%$, without compromising target coverage or normal tissue sparing.

Keywords - proton therapy, robust optimization, minimax 


\section{${ }_{47}$ I. Introduction}

The superior dose distributions produced by intensity-modulated proton therapy (IMPT) indicate a potential for improved patient outcome as compared to conventional X-ray radiotherapy. ${ }^{1,2,3}$ However, it is of critical importance that the IMPT treatment plan is made sufficiently robust in order to prevent an unacceptable deterioration of the treatment at the moment of delivery. Successful treatment planning strategies must therefore take into account treatment uncertainties such as tumor motion, setup and range errors. ${ }^{4,5,6,7}$ In proton therapy treatment planning, the most effective way of handling these uncertainties is to simulate them during the plan optimization process. This approach has led to the development of robust optimization algorithms which provide an alternative to more conventional margin-based approaches. ${ }^{8,9,10,11}$

In general, the different robust optimization algorithms can be classified into two main groups: (1) probabilistic (or stochastic) optimization and (2) worst-case robust optimization. ${ }^{12,13}$ Both groups aim at covering treatment uncertainties by simulating a discrete set of treatment uncertainty scenarios (i.e., realizations of specific combinations of treatment errors). However, the algorithms differ in the way in which the objective function is minimized. Probabilistic optimization algorithms minimize the expected value of the objective function. In contrast, in worst-case robust optimization, the worst-case scenario (the one with the highest objective function value) is chosen, at each iteration, to minimize the objective function.

In this study, we focus on worst-case robust optimization. Different approaches for worst-case robust optimization have been proposed, depending on the way the worst-case scenario is defined. For instance, in voxel-wise worst-case optimization, the worst-case scenario is defined by considering the worst-case value for each individual voxel, among all scenarios (i.e., high dose in organ-at-risk voxels and low dose in the target voxels). ${ }^{8,9}$ However, this approach results in a non-physical and potentially overly conservative solution. ${ }^{10,14}$ For this reason, Fredriksson et. al introduced the so-called 'minimax' optimization where, for each uncertainty scenario, the objective function is computed for all voxels simultaneously. ${ }^{10}$ Minimax optimization for IMPT treatment plans have shown to yield clinically acceptable target coverage, in the presence of treatment uncertainties, for a variety of tumor locations. ${ }^{15,16}$ The main drawback of both minimax and voxel-wise worst-case optimization is their computationally expensive nature, both in terms of the plan computation time and memory consumption. This is due to the following two main issues: first, dose-influence 
matrices must be computed and stored for each treatment uncertainty scenario and second, dose distributions must be re-evaluated, at each iteration, for all scenarios defined within the uncertainty set. Because the uncertainty sources (such as tumor motion, setup error and range errors) are usually handled in a mutually independent way, moving targets are especially resource demanding, as their increased number of uncertainty sources amount to a large number of scenarios. This limits the potential of minimax optimization as a standard clinical tool and prevents its applicability in online-adaptive workflows. ${ }^{17}$

An example of an approach that aims at reducing the plan computation time is to reduce the number of uncertainty scenarios, with the goal of limiting the number of scenario evaluations during optimization. To this end, in a previous study, a planning strategy was proposed that pre-selects a reduced set of relevant uncertainty scenarios, resulting in a significant plan computation time gain. ${ }^{18}$ In contrast, in this study, the full pre-defined uncertainty set is maintained, but we propose an approximate 'dynamic' minimax algorithm that deals with the inherently long optimization time of the conventional minimax optimization algorithm. We focus on accelerating minimax optimization by considering only a reduced set of scenarios, selected from the full uncertainty set. This reduced set is then dynamically updated throughout the optimization process, in order to retain only those scenarios that are mostly active in guiding the optimization solution. The present study aims to address the feasibility of this dynamic minimax optimization and analyses the time gain with respect to conventional minimax. In order to illustrate the proposed method, six lung cancer patients with various tumor sizes and motions are used.

\section{Material and Methods}

In this section, first, the conventional minimax optimization algorithm is formalized, followed by a detailed presentation of the proposed dynamic minimax optimization algorithm. Afterwards, an overview is given of the optimization software and patient data used for the testing and evaluation of the respective methods. 


$$
\begin{aligned}
& \min _{w} \max _{s}\left\{f_{o b j}(d(w, s))\right\} \\
& \text { s.t. }\left\{\begin{array}{l}
w \geq 0 \\
s \in S,
\end{array}\right.
\end{aligned}
$$

with $f_{o b j}$ as the objective function, $d$ the dose distribution and $w$ the optimization variables (i.e., the spot weights) which are constrained to allow only positive solutions. The conventional minimax algorithm is characterized by the following three steps performed at each iteration of optimization: (1) the dose distribution is computed for all scenarios $s$ in $S$ with the objective function $f_{o b j}$ evaluated in each of the scenarios, (2) the worst-case scenario is selected as the scenario in which the objective function attains its highest value and (3) the spot weights $w$ are updated by minimizing the objective function of the current worst-case scenario.

\section{II.B. Dynamic Minimax Optimization}

The proposed algorithm differs from the conventional minimax optimization algorithm by decomposing the pre-defined uncertainty set $S$ into two scenario pools: (1) an 'active pool' $S_{A}$ of candidate-worst scenarios (the pool size of $S_{A}$ is denoted as $N_{A}$ ) and (2) a 'dead pool' $S_{D}$ containing the leftover scenarios (the number of dead pool scenarios is denoted as $N_{D}$ ). Hence, the union of both pools is equal to $S\left(S_{A} \cup S_{D}=S\right)$. From this point onward, we denote the active pool scenarios and dead pool scenarios as 'active scenarios' and 'dead scenarios', respectively. The idea is to identify the scenarios that are mostly used in guiding the optimization solution and include these scenarios into the active pool $S_{A}$. Subsequently, at each iteration, only the active scenarios $\left(s \in S_{A}\right)$ are considered. Hence, the dynamic minimax algorithm can be re-formulated as follows:

$$
\begin{aligned}
& \min _{w} \max _{s}\left\{f_{o b j}(d(w, s))\right\} \\
& \text { s.t. }\left\{\begin{array}{l}
w \geq 0 \\
s \in S_{A} .
\end{array}\right.
\end{aligned}
$$

The active scenarios $\left(s \in S_{A}\right)$ are probabilistically selected, based on an auxiliary variable $P$, the so-called 'acceptance probability set' $P=\left\{P_{s} \mid s \in S\right\}$ which associates with each scenario the 
probability that it might be evaluated as the worst case. ${ }^{1} P$ serves a similar role to the acceptance probability function commonly found in simulated annealing optimization schedules. ${ }^{19}$ Because $P$ plays a key role in the dynamic minimax algorithm, we explain in the following two paragraphs (1) how $P$ is updated over time and (2) how active scenarios are subsequently selected from $P$.

\section{II.B.1. Acceptance probability set $P$}

At each iteration, the acceptance probability $P$ is updated by performing two steps. In the first step, the value $P_{s}$ of the current worst-case scenario $\left(s=s_{\text {worst }}\right)$ is incremented by a factor $\alpha(t)$ :

$$
P_{s}(t)=P_{s}(t-1)+\alpha(t) \text { if } s=s_{w o r s t},
$$

followed by a re-normalization of $P$ :

$$
P_{s}(t)=P_{s}(t) \times \frac{1}{1+\alpha(t)} \forall s \in S
$$

with $t$ the iteration number and $\alpha(t)$ a global time-varying parameter. Following simulated annealing optimization, $\alpha(t)$ is chosen to decay over time and is defined as $\alpha(t)=1 / t$. In doing so, $P$ gradually reduces its sensitivity to fluctuations in the optimization process (so-called optimization noise). In the second step, the values $P_{s}$ of the current dead scenarios $\left(s \in S_{D}\right)$ are incremented by a factor $\alpha(t) / N_{D}$ :

$$
P_{s}(t)=P_{s}(t-1)+\frac{\alpha(t)}{N_{D}} \forall s \in S_{D}(t),
$$

again followed by a re-normalization of $P$ :

$$
P_{s}(t)=P_{s}(t) \times \frac{1}{1+\alpha(t)} \forall s \in S .
$$

Step 2 is performed in order to add the possibility that yet unconsidered (i.e., dead) scenarios may become active at a later point in the optimization. In Eq. $4, \alpha(t)$ is weighted by the size of the dead pool, ensuring that a worst-case evaluation (Eq. 2) weights more than its absence from the active pool. The re-normalization steps of $P$ (Eqs. 3 and 5) are necessary to maintain at all times, a total probability mass of 1 (see Section II.B.2.). Additionally, they serve to effectively reduce the values of inactive scenarios (that is, scenarios present in the active pool but not contributing to the optimization) so that these can eventually be discarded.

\footnotetext{
${ }^{1}$ It must be noted that this scenario 'probability' $P_{s}$ does not bear a resemblance with the uncertainty probability of the scenario, typically used in probabilistic optimization.
} 


\section{II.B.2. Active pool $S_{A}$}

Throughout the optimization process, the active pool scenarios are selected by randomly sampling (without replacement), $N_{A}$ number of scenarios according to their probabilities specified in $P$. In other words, each scenario can only be drawn once, with the probabilities in $P$ normalized after each draw, in order to maintain a probability mass of 1 .

In practice, the active pool is updated at discrete points during the optimization process (in our case at an iteration interval of $\Delta t=10$ ). At the start, $P$ is initialized by assigning a uniform probability distribution with no scenarios left unconsidered (i.e., all scenarios $s \in S$ are evaluated). After the first active pool update, the active pool size is set to its reduced size and active scenarios will be selected using the method described above. Furthermore, because some planning objectives (typically the OAR dose constraints) are evaluated in the nominal scenario only, the nominal scenario is always included active pool throughout the entire optimization process.

In general, the dynamic minimax algorithm is characterized by the size of the active pool $N_{A}$, which is a user-defined parameter. In Section III., we will investigate how the choice of $N_{A}$ influences the resulting optimization process.

\section{II.C. Optimization Software}

The proposed dynamic minimax algorithm was implemented in the open-source treatment planning system MIROpt, coded in Matlab (MathWorks, Natick, United States). ${ }^{20,21}$ MIROpt uses the open-source Monte Carlo dose engine MCsquare for its dose calculations (MCsquare has been validated for clinical practice from commissioning measurements). ${ }^{22,23}$ Dose calculations are performed with $10^{5}$ ions per spot on a $2 \times 2 \times 2 \mathrm{~mm}^{3}$ dose grid and the spot weights are optimized using a gradient descent algorithm. Constraints on the optimization variable (spot weights $w$ ) are handled by a simple projection method, that is, negative values of $w$ are projected to the admissible solution space by setting their values to zero. In order to compare the optimization times of the different optimization algorithms, the maximum number of iterations obtained from the conventional minimax optimization is subsequently used in the dynamic minimax optimizations.

A quadratic objective function is used to penalize deviations from the pre-defined treatment planning objectives. As would be performed conventionally in clinical practice, only the target planning objectives were handled robustly (i.e., evaluated for all considered uncertainty scenarios) 
whilst the OAR objectives were evaluated in the nominal scenario only. Plan optimization was performed on a 256GB RAM system with a 2x8 Core Intel Xeon processor (E5-2667 v3) @3.20 $\mathrm{GHz}$.

For the dynamic minimax optimizations, both the objective functions in the 'approximate' (= proxy) worst-case scenario (i.e., the worst-case scenario evaluated only for the active pool scenarios) and the 'exact' worst-case scenario (i.e., evaluated for all uncertainty scenarios) will be reported in the results Section III. The former is, from this point onward, denoted as the 'proxy worst-case scenario' and the latter as the 'exact worst-case scenario'. Generally, in the dynamic minimax optimization, the exact worst-case scenario is unavailable as not all uncertainty scenarios are evaluated at each iteration. However, in order to compare the different methods, additional dynamic minimax optimizations are performed where all uncertainty scenarios are evaluated, storing the objective function in the exact worst-case scenario as well.

\section{II.D. Robustness Evaluation}

The robustness of all resulting plans was evaluated with MCsquare, by using a comprehensive approach in which the dose distribution is recomputed on a set of 250 treatment error evaluation scenarios. These evaluation scenarios include effects of systematic setup errors, range errors and respiratory motion. ${ }^{24}$ Setup errors and range errors are sampled from normal distributions with a standard deviation of $2 \mathrm{~mm}$ and $1.6 \%,{ }^{25}$ respectively, whilst respiratory motion is modeled by recomputing the dose on each breathing phase $\mathrm{CT}$ and accumulating the dose on the reference (timeaveraged mid-position (MidP)) CT. ${ }^{26}$ A $90 \%$ confidence interval is generated in the dosimetric space by discarding the $10 \%$ worst scenarios (based on the target $\mathrm{D}_{95}$ ) of the above-mentioned 250 error scenarios. ${ }^{24}$ The number of protons is selected in order to reach a statistical uncertainty of $1 \%$.

For the dosimetric plan evaluations, the target DVH metrics (CTV $\mathrm{D}_{95}$ and CTV $\mathrm{D}_{5}$ ) are calculated in the worst-case evaluation scenario, i.e., the scenario where the lowest target coverage is realized (based on CTV $\mathrm{D}_{95}$ ), within the $90 \%$ confidence interval generated using the method mentioned above. In the results section (Section III.), this worst-case evaluation scenario will be referred to as the 'tested worst-case scenario', in order to draw a distinction between the worst-case scenarios used throughout the optimization process (i.e., the proxy and exact worst-case scenarios) and the worst-case scenario used for the robustness evaluation (i.e., the tested worst-case scenario). The CTV bandwidths (BW) at the $\mathrm{D}_{95}$ and $\mathrm{D}_{5}$ dose levels, are calculated within the same $90 \%$ 
confidence interval. In other words, the BW represents the difference in dose between the tested best-case and tested worst-case scenario, at a given dose level. The OAR DVH metrics will be calculated in the nominal scenario only, meaning that the dose distribution is recomputed on the nominal planning CT with a statistical uncertainty of $1 \%$.

\section{II.E. Patient Cases}

Six lung tumor patients were chosen to test the proposed optimization algorithm, as their treatment planning typically involves a large number of optimization scenarios, causing long plan optimization times. Patient data were characterized by a 4D-CT image set, binned in ten breathing phases, evenly spaced in time. All patients presented a single tumor volume, delineated on the MidPCT. The main features of the patient cohort are summarized in Table 1. All patients had a dose prescription of 60 Gy to the clinical-target-volume (CTV) with target coverage considered acceptable if $95 \%$ of the CTV received more than $95 \%$ of the prescribed dose (= 57 Gy), whilst no more than $5 \%$ of the CTV received over $105 \%$ of the prescribed dose (=63 Gy), for the worst-case scenario.

All treatment plans used the MidP-CT as the nominal planning CT which was created with the open-source platform OpenReggui. ${ }^{26,27}$ Treatment plans were optimized using uncertainty scenarios that contain setup errors, range errors and respiratory motion. Similar to other studies, uncertainty parameters were chosen as combinations of $5 \mathrm{~mm}$ setup errors in the three directions (left-right, anterior-posterior and superior-inferior), $\pm 3 \%$ range error and maximum inhale and exhale breathing phases, generating an uncertainty set of 63 scenarios ( $=7$ setup error scenarios $\times 3$ range error scenarios $\times 3$ breathing phases). ${ }^{6,8,10,28}$ Setup and range errors are modeled by rigidly shifting the $\mathrm{CT}$ image and uniformly scaling the CT mass densities (obtained from the CT image), respectively. All treatment plans were designed using a configuration of three co-planar beams, delivered via IMPT with the pencil beam scanning (PBS) technique (see Table 1). 
Table 1: Patient characteristics.

\begin{tabular}{|c|c|c|c|c|c|c|}
\hline \multirow[t]{2}{*}{ Patient } & \multirow{2}{*}{$\begin{array}{c}\text { CTV size } \\
{\left[\mathrm{cm}^{3}\right]}\end{array}$} & \multicolumn{3}{|c|}{ Motion Amplitude } & \multirow[t]{2}{*}{ Tumor position } & \multirow[t]{2}{*}{ Beam angles } \\
\hline & & $\begin{array}{c}\mathrm{LR} \\
{[\mathrm{mm}]}\end{array}$ & $\begin{array}{c}\mathrm{AP} \\
{[\mathrm{mm}]}\end{array}$ & $\begin{array}{c}\mathrm{SI} \\
{[\mathrm{mm}]}\end{array}$ & & \\
\hline P1 & 152.6 & 4.2 & 2.1 & 3.1 & RML & $0,270,310$ \\
\hline P2 & 107.7 & 3.1 & 2.9 & 3.7 & LLL & $90,135,180$ \\
\hline P3 & 41.3 & 1.4 & 2.9 & 0.8 & RUL & $180,225,270$ \\
\hline P4 & 70.3 & 0.8 & 1.2 & 0.5 & LUL & $90,135,180$ \\
\hline P5 & 109.6 & 2.2 & 1.8 & 6.6 & RUL & $180,225,270$ \\
\hline P6 & 249.7 & 2.1 & 2.5 & 10.6 & RLL & $180,225,270$ \\
\hline
\end{tabular}

Tumor motion amplitude (in left-right (LR), anterior-posterior $(A P)$ and superior-inferior (SI) directions). Tumor positions (right-middle lobe (RML), left-lower lobe (LLL), rightupper lobe (RUL), right-lower lobe (RLL) and left-upper lobe (LUL)).

\section{Results}

In this section, the performance of dynamic minimax optimization algorithm is compared to the conventional minimax optimization. As mentioned in Section II., the conventional minimax algorithm evaluates, at each iteration, all 63 scenarios in the uncertainty set. Because the dynamic minimax is characterized by the parameter $N_{A}$, we present the results for two different choices of $N_{A}$, that is, $N_{A}=15$ and a more extreme case of $N_{A}=5$. The performance of the optimizations will be assessed by the achieved time-gain and the resulting plan quality. The plan quality is measured first, according to the value of the worst-case objective function value throughout the optimization process (so-called optimization curve) and second, from the dosimetric metrics (target coverage, robustness and OAR sparing) obtained after performing comprehensive robustness evaluations (see Section II.D.).

\section{III.A. Optimization Data Results}

Table 2 reports the plan optimization times, together with the final (worst-case) objective function value $f$. For the dynamic minimax optimization, the final objective function values $f_{\text {proxy }}$ and $f_{\text {exact }}$ are reported, which represent the objective function evaluated in the proxy worst-case and the exact worst-case scenario, respectively (see Section II.C.). Because the conventional minimax optimization evaluates, by default, all uncertainty scenarios, its final worst-case objective function 
Table 2: Plan optimization time, final worst-case objective function values $f_{\text {proxy }}$ (evaluated only for the active pool scenarios) and $f_{\text {exact }}$ (evaluated for all scenarios). Plans of each patient (P1-6) were obtained using the conventional minimax optimization (Ref.) and dynamic minimax optimization algorithms with pool sizes of $N_{A}=5$ and $N_{A}=15$. The average time reductions (in \%) are reported at the bottom.

\begin{tabular}{lccc|ccc|cc}
\hline & \multicolumn{3}{c}{ Optimization time $[\mathrm{min}]$} & \multicolumn{3}{c}{ Final $f_{\text {exact }}$} & \multicolumn{3}{c}{ Final $f_{\text {proxy }}$} \\
& Ref. & $N_{A}=15$ & $N_{A}=5$ & Ref. & $N_{A}=15$ & $N_{A}=5$ & $N_{A}=15$ & $N_{A}=5$ \\
\hline P1 & 513 & 170 & 85 & 1.55 & 1.45 & 1.27 & 1.43 & 1.08 \\
P2 & 396 & 142 & 72 & 0.74 & 0.63 & 0.69 & 0.61 & 0.55 \\
P3 & 167 & 47 & 22 & 1.96 & 1.79 & 2.24 & 1.78 & 1.63 \\
P4 & 219 & 79 & 32 & 2.97 & 2.57 & 3.02 & 2.50 & 1.71 \\
P5 & 409 & 152 & 83 & 1.02 & 0.98 & 1.07 & 0.93 & 0.73 \\
P6 & 758 & 213 & 107 & 6.0 & 5.3 & 4.8 & 5.3 & 4.4 \\
\hline$\Delta$ Avg. & & $-67 \%$ & $-84 \%$ & & & & & \\
\hline
\end{tabular}




\section{III.B. Dosimetric Results}

Table 3 and Table 4 show the target and OAR DVH metrics for the obtained treatment plans. Target coverage metrics $\left(\mathrm{D}_{95}\right.$ and $\left.\mathrm{D}_{5}\right)$ are calculated in the tested worst-case scenario whilst the OAR metrics are calculated in the nominal scenario only (see Section II.D.). Furthermore, the average difference between the value in the reference plan (obtained using conventional minimax optimization algorithm) with plans optimized using the dynamic minimax algorithms is shown for each metric.

On average, equal target coverage (worst-case $\mathrm{CTV} \mathrm{D}_{95}$ ) is obtained between the conventional minimax and $N_{A}=15$ dynamic minimax optimization. The $N_{A}=5$ dynamic minimax optimization improved worst-case CTV $\mathrm{D}_{95}$ slightly by $0.2 \mathrm{~Gy}$, on average, with respect to the reference plans. OAR dose is similar between all studied plans (average difference of mean lung dose of only 0.2 Gy and 0.4 Gy between the conventional minimax and $N_{A}=15$ and $N_{A}=5$ dynamic minimax optimizations, respectively and difference in mean esophagus dose of -0.1 Gy and 0.1 Gy, respectively).

Fig. 2 displays the dose distribution together with the corresponding DVHs for each optimization method. Results indicate similar dose profiles between all plans with isodose lines that nearly coincide. This similarity translates to DVHs that have a similar sensitivity to the treatment errors (indicated by the CTV BWs in Table 3) and matching OAR DVH curves. 
Table 3: Target coverage metrics (CTV $\mathrm{D}_{95}$ and $\left.\mathrm{D}_{5}\right)$ and robustness metrics (CTV bandwidth (BW) at the $\mathrm{D}_{95}$ and $\mathrm{D}_{5}$ dose level) for plans of all patients (P1-6), obtained using conventional minimax (Ref.) and dynamic minimax optimization with pool sizes of $N_{A}=5$ and $N_{A}=15$. CTV $\mathrm{D}_{95}$ and $\mathrm{D}_{5}$ are computed in the tested worst-case scenario.

\begin{tabular}{|c|c|c|c|c|c|c|}
\hline & \multicolumn{6}{|c|}{ CTV } \\
\hline & \multicolumn{3}{|c|}{ Worst-case $D_{95}[\mathrm{~Gy}]$} & \multicolumn{3}{|c|}{ Worst-case $\mathrm{D}_{5}[\mathrm{~Gy}]$} \\
\hline & Ref. & $N_{A}=15$ & $N_{A}=5$ & Ref. & $N_{A}=15$ & $N_{A}=5$ \\
\hline P1 & 57.0 & 56.9 & 57.3 & 62.8 & 62.4 & 62.4 \\
\hline P2 & 57.6 & 57.6 & 57.3 & 61.8 & 61.8 & 61.7 \\
\hline P3 & 58.0 & 57.9 & 58.5 & 62.6 & 62.6 & 61.9 \\
\hline P4 & 58.2 & 58.3 & 58.6 & 62.1 & 62.1 & 62.4 \\
\hline P5 & 58.3 & 58.4 & 58.5 & 61.7 & 61.7 & 61.6 \\
\hline P6 & 57.2 & 57.4 & 57.2 & 64.2 & 63.6 & 63.6 \\
\hline \multirow[t]{3}{*}{$\Delta \mathrm{Avg}$. } & & 0.0 & +0.2 & & -0.2 & -0.3 \\
\hline & \multicolumn{3}{|c|}{$\mathrm{BW}$ at $\mathrm{D}_{95}[\mathrm{~Gy}]$} & \multicolumn{3}{|c|}{$\mathrm{BW}$ at $\mathrm{D}_{5}[\mathrm{~Gy}]$} \\
\hline & Ref. & $N_{A}=15$ & $N_{A}=5$ & Ref. & $N_{A}=15$ & $N_{A}=5$ \\
\hline P1 & 1.9 & 1.8 & 1.5 & 1.1 & 0.9 & 1.0 \\
\hline P2 & 1.1 & 0.9 & 0.9 & 0.9 & 0.8 & 0.8 \\
\hline P3 & 0.6 & 0.8 & 0.3 & 1.1 & 1.2 & 1.4 \\
\hline P4 & 0.6 & 0.6 & 0.4 & 1.0 & 1.0 & 1.4 \\
\hline P5 & 0.6 & 0.5 & 0.4 & 0.6 & 0.7 & 0.5 \\
\hline P6 & 1.6 & 1.5 & 1.7 & 1.6 & 1.6 & 1.6 \\
\hline$\Delta$ Avg. & & -0.1 & -0.2 & & 0.0 & +0.1 \\
\hline
\end{tabular}

Table 4: Organ-at-risk DVH metrics (lung, esophagus and heart) for plans of all patients (P1-6), obtained using conventional minimax (Ref.) and dynamic minimax optimization with pool sizes of $N_{A}=5$ and $N_{A}=15$. Metrics have been computed in the nominal scenario.

\begin{tabular}{|c|c|c|c|c|c|c|c|c|c|c|c|c|}
\hline & \multicolumn{6}{|c|}{ Lung } & \multirow{2}{*}{\multicolumn{3}{|c|}{$\begin{array}{c}\text { Esophagus } \\
\mathrm{D}_{\text {mean }}[\mathrm{Gy}]\end{array}$}} & \multirow{2}{*}{\multicolumn{3}{|c|}{$\begin{array}{c}\text { Heart } \\
\mathrm{V}_{40}[\%]\end{array}$}} \\
\hline & \multicolumn{3}{|c|}{$\mathrm{V}_{20}[\%]$} & \multicolumn{3}{|c|}{$\mathrm{D}_{\text {mean }}[\mathrm{Gy}]$} & & & & & & \\
\hline & Ref. & $N_{A}=15$ & $N_{A}=5$ & Ref. & $N_{A}=15$ & $N_{A}=5$ & Ref. & $N_{A}=15$ & $N_{A}=5$ & Ref. & $N_{A}=15$ & $N_{A}=5$ \\
\hline P1 & 26.4 & 26.5 & 28.3 & 13.5 & 13.6 & 14.1 & 2.0 & 2.0 & 2.1 & 3.2 & 3.3 & 3.4 \\
\hline P2 & 26.9 & 27.2 & 27.8 & 13.5 & 13.6 & 13.9 & 5.4 & 5.5 & 5.7 & 3.8 & 3.9 & 4.0 \\
\hline P3 & 13.4 & 13.4 & 13.6 & 7.0 & 7.0 & 7.2 & 4.8 & 4.8 & 5.0 & 0.0 & 0.0 & 0.0 \\
\hline P4 & 19.0 & 19.1 & 19.4 & 9.7 & 9.8 & 10.0 & 2.1 & 2.1 & 2.2 & 0.0 & 0.0 & 0.0 \\
\hline P5 & 21.9 & 22.1 & 22.4 & 10.6 & 10.8 & 10.9 & 7.9 & 8.0 & 8.3 & 1.1 & 1.2 & 1.2 \\
\hline P6 & 30.0 & 31.6 & 31.6 & 16.0 & 16.6 & 16.6 & 20.3 & 19.7 & 19.7 & 3.3 & 3.4 & 3.4 \\
\hline$\Delta$ Avg. & & +0.4 & +0.9 & & +0.2 & +0.4 & & -0.1 & +0.1 & & +0.1 & +0.1 \\
\hline
\end{tabular}



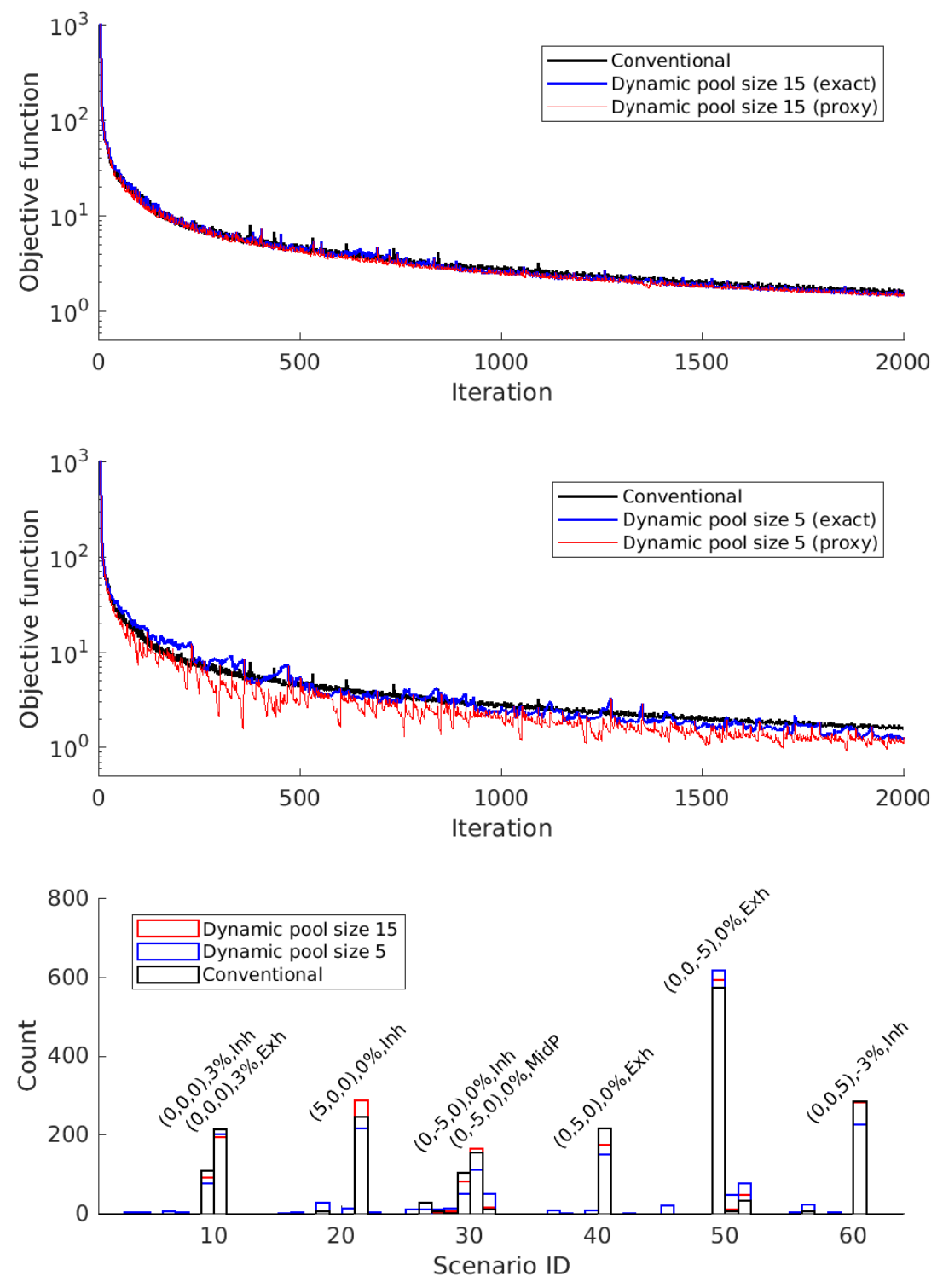

Figure 1: Comparison of conventional minimax and dynamic minimax optimizations (results of patient P1 are displayed). The top and middle panels show the progression of the (worstcase) objective function value throughout the optimization (top: pool size of $N_{A}=15$ and middle: pool size of $N_{A}=5$ ). For the dynamic minimax optimization, the proxy worst-case objective function $f_{\text {proxy }}$ is displayed in red, whilst the exact worst-case objective function $f_{\text {exact }}$ is displayed in blue. The bottom panel shows the number of iterations (= counts) that each scenario is evaluated as the worst case. The magnitude of the uncertainties is shown for the most counted scenarios. The uncertainties are displayed as follows: setup error $(x, y, z)$ in $\mathrm{mm}$ in the left-right $x$, anterior-posterior $y$ and superior-inferior $z$ directions, range error and breathing phase (MidP, max inhale or max exhale). 

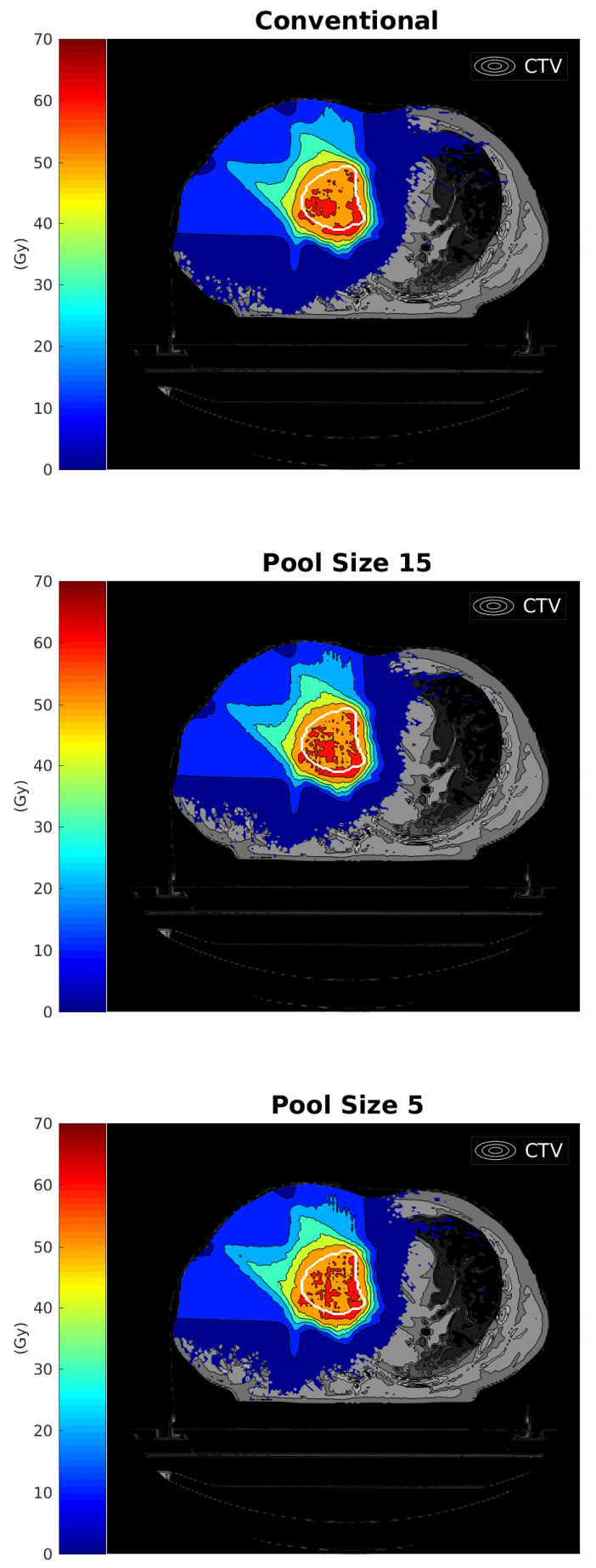
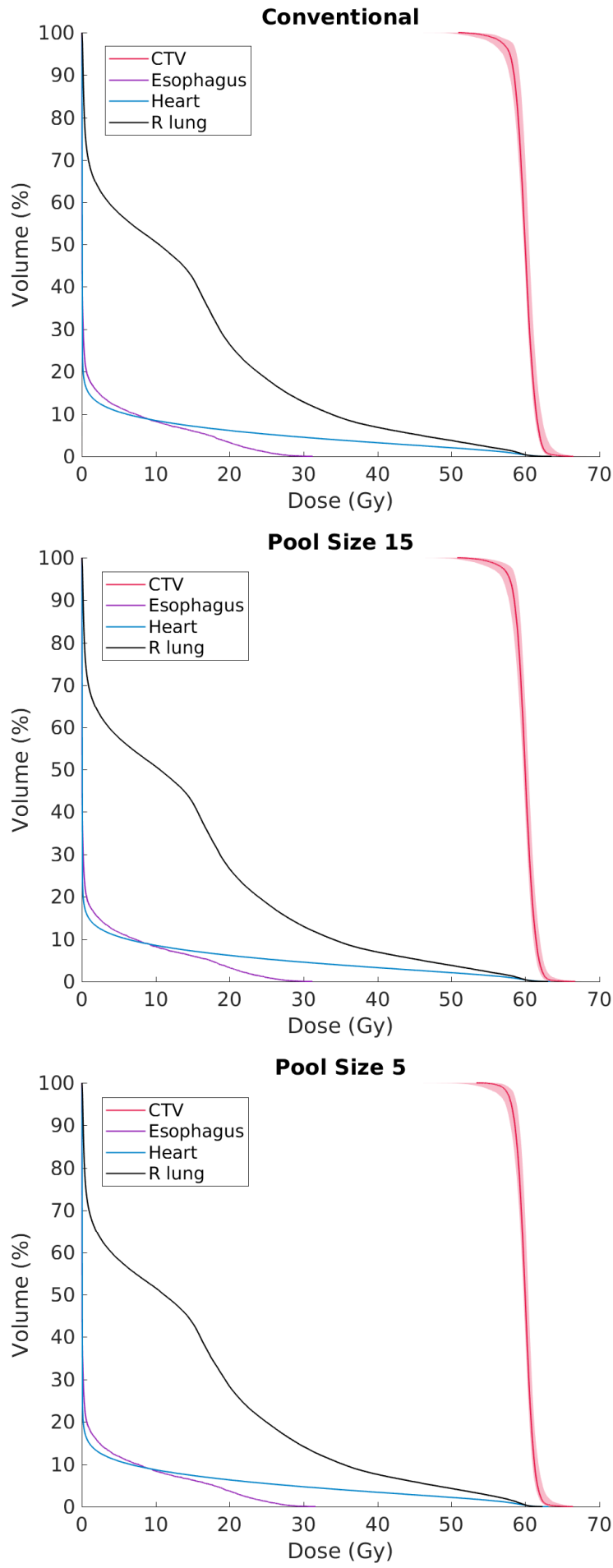

Figure 2: The left column shows the dose distributions for plans obtained using conventional minimax optimization and dynamic minimax optimization with pool sizes of $N_{A}=5$ and $N_{A}=15$ for patient P1. In each figure, the CTV is indicated in white. The right column shows the corresponding DVHs with the CTV-DVH band representing the evaluations in the considered evaluation scenarios (see Section II.D.). 


\section{Discussion}

In minimax optimization, only the current worst-case scenario is used to guide the optimization solution. In the meantime, as noted by Fredriksson et. $a l,{ }^{14}$ minimax algorithms tend to neglect so-called 'easy' scenarios, that is, scenarios where there is little conflict between organ sparing and target coverage in the objective function. Hence, a substantial amount of computation time and resources are potentially wasted on scenario evaluations that are rarely the worst case. Fig. 1 (bottom) illustrates this feature of minimax optimization by showing that the optimizer only accesses a fraction of the full uncertainty set in order to reach its final solution. This suggests that the majority of scenarios produce either comparable dose distributions or produce dose distributions where the planning objectives are consistently well respected. Fredriksson argues that disregarding 'easy' scenarios is one of the main disadvantages of the minimax algorithm when comparing it to other classes of robust optimization algorithms. ${ }^{14}$ In fact, it is exactly this drawback that the dynamic minimax algorithm attempts to address. By relying on the sparsity of active scenarios in the solution space, fewer scenarios are needed whilst still preserving most of the information of the full problem. In doing so, the computational cost of an iteration is significantly reduced (in other words, the number of scenario evaluations performed at each iteration is reduced), resulting in an accelerated optimization process (a time gain of up to $84 \%$ is obtained).

The optimization curves in Section III.A. show that by reducing the size of the active pool $N_{A}$, the optimization noise level increases. Fundamentally, worst-case robust optimization is inherently a noisy optimization process. This is explained by the fact that different optimization scenarios are used throughout the optimization as a result of the discontinuous max operator (see Eq. 1). Additionally, the projection method (see Section II.C.), to handle constraints on the optimization variables (the spot weights), also adds noise to the optimization. In addition to the above-mentioned noise sources, the dynamic minimax algorithm, will add optimization noise by regularly changing the possible optimization scenarios throughout the optimization process. This effect will be more pronounced for smaller active pool sizes, which change their composition more frequently. The additional noise level produced by the dynamic minimax algorithm is exemplified in Fig. 1 (bottom). As shown, optimizations with smaller pool sizes will explore an increased number of scenarios in the solution space. By increasing the pool size slightly (to $N_{A}=15$ ), the noise is reduced to a level comparable in magnitude to the conventional minimax optimization. However, as the results of Section III.B. indicate, the increased optimization noise level does not harm the 
final quality of the treatment plans. In fact, results indicate that a noisy optimization trajectory in the solution space might be advantageous in order to further explore and eventually find a better solution; this is an approach commonly employed in simulated annealing and stochastic gradient descent optimization schedules.

The dynamic minimax algorithm was tested for 4D-robust optimization of lung tumor cases with motion. Moving lung tumor cases typically present difficulties in terms of generating robust treatment plans with acceptable plan quality, hence these cases where chosen to test the proposed method. The dynamic minimax optimization could be applied to 3D-robust optimization, however, the time-gain is expected to be less significant since 3D-robust optimization typically uses less uncertainty scenarios for its optimization.

In order to further validate the optimality of the proposed the pool size, the algorithm should be tested for a wider set of patient cases. For instance, in highly complex cases (i.e., large tumor motion with considerable conflicts among the planning objectives), it is recommended to employ a more conservative approach by using a larger the active pool size. This would guarantee that important scenarios are not missed throughout the optimization. Based on the results of the present study, by using a pool size of 15 , almost equal results are obtained as for the conventional minimax whilst still achieving a significant plan optimization time gain of $67 \%$. As a future perspective, instead of a fixed pool size, an adaptive pool size could be considered which could identify the necessary number of active pool scenarios. By adapting the active pool size over time, such an adaptive dynamic minimax algorithm should be able to handle automatically those cases where numerous scenarios contribute equally in the optimization.

It must be noted that this study only focuses on reducing the optimization time and does not deal with other computational aspects (such as the memory consumption) of minimax optimization. In particular, the computation of the beamlet dose-influence matrices gives a large contribution to the overall plan computation time (especially for Monte Carlo-based dose computations). The following solutions exist that can reduce the dose computation time and which could potentially be used in conjunction with the dynamic minimax optimization: first, the number of beamlet doseinfluence matrices can be reduced by performing a pre-selection of relevant uncertainty scenarios, ${ }^{18}$ and second, a hybrid Monte Carlo-pencil beam dose optimizer can be used to accelerate the plan computation time with Monte-Carlo like accuracy. ${ }^{21}$ 
350

\section{Conclusions}

In minimax optimization, the dose distributions must be evaluated for all uncertainty scenarios in order to evaluate their respective objective functions. As a result, the plan optimization time linearly scales with the number of pre-defined uncertainty scenarios. Especially for lung tumor patients, which need a large number of scenarios to robustly optimize their treatment plans, the associated computational burden may cause excessive plan computation times. This issue limits the use of robust optimization in the clinical environment.

In this study, we propose an approximate worst-case robust optimization algorithm that accelerates minimax optimization. The proposed dynamic minimax algorithm relies on the fact that minimax algorithms neglect so-called 'easy' scenarios where there is little conflict among the planning objectives. Therefore, instead of evaluating all scenarios in the pre-defined uncertainty set, only a reduced set of active pool scenarios is considered. Following stochastic annealing optimization schedules, these active scenarios are updated according to a variable called the 'acceptance probability set'. This variable expresses the probability that a scenario might be evaluated as the worst case. By doing so, only the scenarios that are contributing most to the optimization, at that moment, will be retained and accessible in order to guide the optimization solution. The proposed method was applied to 4D-robust minimax optimization and tested for six moving lung tumor cases. Results show that, on average, an optimization time gain of up to $84 \%$ is achieved without compromising either target robustness or normal tissue sparing.

\section{Acknowledgements}

Gregory Buti is supported by the Télévie Grant from the Belgian 'Fonds National pour la Recherche Scientifique' F.R.S-FNRS (Grant No. 7453918F). Computational resources have been provided by the supercomputing facilities of the Universite Catholique de Louvain (CISM/UCL) and the Consortium des Équipements de Calcul Intensif en Fédération Wallonie Bruxelles (CÉCI) funded by the F.R.S.-FNRS under convention 2.5020.11. Kevin Souris is funded by the Walloon region (MECATECH/BIOWIN, Grant No. 8090). Ana M. Barragán Montero is funded by the Walloon region (PROTHERWAL/CHARP, Grant No. 7289). Marie Cohilis is supported by the Télévie Grant from the F.R.S-FNRS (Grant No. 7450517F). John A. Lee is a Senior Research Associate with the F.R.S.-FNRS. 


\section{References}

1 A. Elhammali, P. Blanchard, A. Yoder, Z. Liao, X. Zhang, X. R. Zhu, P. K. Allen, M. Jeter, J. Welsh, and Q.-N. Nguyen, Clinical outcomes after intensity-modulated proton therapy with concurrent chemotherapy for inoperable non-small cell lung cancer, Radiotherapy and Oncology 136, 136-142 (2019).

2 N. Nakamura, K. Hotta, S. Zenda, H. Baba, S. Kito, T. Akita, A. Motegi, H. Hojo, M. Nakamura, R. V. Parshuram, M. Okumura, and T. Akimoto, Hypofractionated proton beam therapy for centrally located lung cancer, Journal of Medical Imaging and Radiation Oncology 63, $552-556(2019)$

3 R. M. Hoshina, T. Matsuura, K. Umegaki, and S. Shimizu, A Literature Review of Proton Beam Therapy for Prostate Cancer in Japan, Journal of Clinical Medicine 8, 48 (2019).

4 P. C. Park, J. P. Cheung, X. R. Zhu, A. K. Lee, N. Sahoo, S. L. Tucker, W. Liu, H. Li, R. Mohan, L. E. Court, and L. Dong, Statistical Assessment of Proton Treatment Plans Under Setup and Range Uncertainties, International Journal of Radiation Oncology*Biology*Physics 86, 1007-1013 (2013).

5 S. Brousmiche, K. Souris, J. O. de Xivry, J. A. Lee, B. Macq, and J. Seco, Combined influence of CT random noise and HU-RSP calibration curve nonlinearities on proton range systematic errors, Physics in Medicine \& Biology 62, 8226-8245 (2017).

6 A. J. Lomax, Intensity modulated proton therapy and its sensitivity to treatment uncertainties 1: the potential effects of calculational uncertainties, Physics in Medicine and Biology 53, 1027-1042 (2008).

7 A. J. Lomax, Intensity modulated proton therapy and its sensitivity to treatment uncertainties 2: the potential effects of inter-fraction and inter-field motions, Physics in Medicine and Biology 53, 1043-1056 (2008).

8 W. Liu, X. Zhang, Y. Li, and R. Mohan, Robust optimization of intensity modulated proton therapy, Medical Physics 39, 1079-1091 (2012).

9 D. Pflugfelder, J. J. Wilkens, and U. Oelfke, Worst case optimization: a method to account for uncertainties in the optimization of intensity modulated proton therapy, Physics in Medicine and Biology 53, 1689-1700 (2008). 
10 A. Fredriksson, A. Forsgren, and B. Hårdemark, Minimax optimization for handling range and setup uncertainties in proton therapy, Medical Physics 38, 1672-1684 (2011).

11 J. Unkelbach, T. Bortfeld, B. C. Martin, and M. Soukup, Reducing the sensitivity of IMPT treatment plans to setup errors and range uncertainties via probabilistic treatment planning, Medical Physics 36, 149-163 (2008).

12 A. Fredriksson, A characterization of robust radiation therapy treatment planning methodsfrom expected value to worst case optimization, Medical Physics 39, 5169-5181 (2012).

13 J. Unkelbach, M. Alber, M. Bangert, R. Bokrantz, T. C. Y. Chan, J. O. Deasy, A. Fredriksson, B. L. Gorissen, M. van Herk, W. Liu, H. Mahmoudzadeh, O. Nohadani, J. V. Siebers, M. Witte, and H. Xu, Robust radiotherapy planning, Physics in Medicine \& Biology 63, 22TR02 (2018).

14 A. Fredriksson and R. Bokrantz, A critical evaluation of worst case optimization methods for robust intensity-modulated proton therapy planning, Medical Physics 41, 081701 (2014).

15 L. V. van Dijk, R. J. H. M. Steenbakkers, B. ten Haken, H. P. van der Laan, A. A. van 't Veld, J. A. Langendijk, and E. W. Korevaar, Robust Intensity Modulated Proton Therapy (IMPT) Increases Estimated Clinical Benefit in Head and Neck Cancer Patients, PLOS ONE 11, e0152477 (2016).

16 D. Cummings, S. Tang, W. Ichter, P. Wang, J. D. Sturgeon, A. K. Lee, and C. Chang, Fourdimensional Plan Optimization for the Treatment of Lung Tumors Using Pencil-beam Scanning Proton Radiotherapy, Cureus (2018).

17 K. Bernatowicz, X. Geets, A. Barragan, G. Janssens, K. Souris, and E. Sterpin, Feasibility of online IMPT adaptation using fast, automatic and robust dose restoration, Physics in Medicine \& Biology 63, 085018 (2018).

18 G. Buti, K. Souris, A. M. B. Montero, J. A. Lee, and E. Sterpin, Towards fast and robust 4D optimization for moving tumors with scanned proton therapy, Medical Physics (2019).

19 S. Kirkpatrick, C. D. Gelatt, and M. P. Vecchi, Optimization by Simulated Annealing, Science 220, 671-680 (1983).

20 A. M. B. Montero, Miropt - http://www.openmiropt.org/ Accessed November 2019. 


\section{Physics 45, 846-862 (2017).}

hybrid Monte Carlo-Pencil Beam dose algorithm for proton therapy inverse planning, Medical

2

S. Huang, M. Kang, K. Souris, C. Ainsley, T. D. Solberg, J. E. McDonough, C. B. Simone, and L. Lin, Validation and clinical implementation of an accurate Monte Carlo code for pencil beam scanning proton therapy, Journal of Applied Clinical Medical Physics 19, 558-572 (2018).

K. Souris, A. B. Montero, G. Janssens, D. D. Perri, E. Sterpin, and J. A. Lee, Technical Note: Monte Carlo methods to comprehensively evaluate the robustness of $4 \mathrm{D}$ treatments in proton therapy, Medical Physics (2019).

H. Paganetti, Range uncertainties in proton therapy and the role of Monte Carlo simulations, Physics in Medicine and Biology 57, R99-R117 (2012).

M. Wanet, E. Sterpin, G. Janssens, A. Delor, J. A. Lee, and X. Geets, Validation of the mid-position strategy for lung tumors in helical TomoTherapy, Radiotherapy and Oncology 110, 529-537 (2014).

27 28 T. Inoue, J. Widder, L. V. van Dijk, H. Takegawa, M. Koizumi, M. Takashina, K. Usui, C. Kurokawa, S. Sugimoto, A. I. Saito, K. Sasai, A. A. van't Veld, J. A. Langendijk, and E. W. Korevaar, Limited Impact of Setup and Range Uncertainties, Breathing Motion, and Interplay Effects in Robustly Optimized Intensity Modulated Proton Therapy for Stage III Non-small Cell Lung Cancer, International Journal of Radiation Oncology*Biology*Physics 96, 661-669 (2016). 